\title{
Overcoming Infertility in Men with Non-Obstructive and Obstructive form of Azoospermia thought the Procedure Tesa
}

\section{Rudneva S. A. ${ }^{1,2}$, Apryshko V. $P^{1,2}$, Khrabrov T. U. ${ }^{1}$, Mironova A.G ${ }^{1,2}$, Larikova N.U ${ }^{1}$, Mar'ina L. V. ${ }^{1}$, Cheblokova T. A. ${ }^{1}$, Bogomolova A. A. ${ }^{1}$, Zhidkov V. A. ${ }^{1}$, Grechishcheva J. S. ${ }^{1}$, Solnyshkova A. A. ${ }^{1}$, Yakovenko S. A ${ }^{1,2}$}

"Altra Vita" Human Reproduction Clinic, Moscow, Russia.

${ }^{2}$ Lomonosov Moscow State University, Moscow, Russia.

*Corresponding Author: Rudneva S. A, Altra Vita" Human Reproduction Clinic, Moscow, Russia.

\section{Abstract}

In recent years, the range of assisted reproductive technologies has expanded significantly, and many couples with infertility, even with its severe forms, were able to have their own children. 272 married couples with male infertility factor - azoospermia who underwent TESA in the period from January 2016 to July 2020 were examined. It was shown that in $73 \%$ of patients with azoospermia it was possible to obtain sperm. Clinical pregnancy occurred in $47 \%$ of this category of patients, but $17 \%$ of them had a miscarriage. In $33 \%$ of patients, pregnancy did not occur, in 9\% of patients did not have normal embryos. It was shown that during the repeated TESA procedure in $89 \%$ of patients in whom sperm were found for the first time sperm were also found. In $8 \%$ of patients with repeated TESA procedure, no sperm were found. in patients with a negative result of the procedure TESA and in the future no sperm was found. It is shown that the negative result of the procedure TESA cannot be explained by age-related changes in the male body, since most of the men in this category were under the age of 40 years.

Keywords: assisted reproductive technologies, IVF, ICSI, obstructive and non-obstructive azoospermia, TESA, AZF-deletions.

One of the most severe forms of male infertility is azoospermia. This is a pathological condition in which a man's ejaculate completely lacks spermatozoa. Determine it during a series of spermograms, examining under a microscope the sediment from the centrifugation of seminal fluid. The prevalence of azoospermia among men who are married and do not have children is $11-14 \%$ [1]. However, azoospermia should be considered as one of the manifestations of spermatogenesis disorders, rather than as an independent disease. Depending on the origin, azoospermia is divided into obstructive OA, caused by a violation of the permeability of the VAS deferens, and Non-Obstructive NOA (secretory). In OA, sperm are produced in the seminal tubules of the testicles, but as a result of obstruction of the VAS deferens, they do not enter the ejaculate, which may be due to trauma, urogenital infections, operations on the scrotum, as well as some genetically determined syndromes, such as cystic fibrosis. An increased frequency of mutations of the cystic fibrosis gene is noted both in patients with NOA and with a severe form of oligospermia, in which there is a decrease in the volume of ejaculate obtained during ejaculation. Currently, more than 1,800 mutations of the cystic fibrosis gene - CFTR (cystic fibrosis transmembrane conduction regulator) have been identified, about 250 of them may predispose to impaired spermatogenesis and / or fertilizing ability of spermatozoa [2]. NOA is diagnosed when there is a violation of maturation or lack of sperm in the testicular tissue, i.e. testicular insufficiency, which occurs in $10 \%$ of infertile men, and is detected in $60 \%$ of men with azoospermia. The causes of testicular insufficiency can be genetic disorders (sex chromosome abnormalities, deletions, translocations, and mutations in the AZF region of the $\mathrm{Y}$ chromosome (Azoospermia Factor)), cryptorchidism, exposure to radiation, chemotherapy, and toxin poisoning $[3,4]$. In 
Overcoming Infertility in Men with Non-Obstructive and Obstructive form of Azoospermia thought the Procedure Tesa

some cases, there is a combination of spermatogenesis disorders of varying degrees in one or both testicles, and complete or incomplete violations of permeability of the VAS deferens, a common cause of which, as already mentioned above, is mutations of the CFTR cystic fibrosis gene [2]. Another common of these genetic causes of spermatogenesis disorders are deletions (loss of a fragment) of the long arm of the $\mathrm{Y}$ chromosome that capture the AZF locus (azoospermia factor) [4-8]. Deletions at a given locus can be complete, i.e., completely removing one, two, or all three of its three regions (AZFa, AZFb и AZFc ), or partial, i.e., not completely capturing any region [5-7]. Almost all complete AZF deletions are de novo mutations and lead to male infertility due to secretory azoospermia or severe oligozoospermia $[5,6]$. Other genetic disorders that are associated with male infertility are Kleinfelter's syndrome, polymorphisms in the gene (AR/HUMARA), Young's syndrome, characterized by systematic blockage of the testicular ducts; and others [3]. In humans, more than 2,300 genes are involved in spermatogenesis, which causes a pronounced genetic heterogeneity of many forms of male infertility [6-9].

Until recently, patients with azoospermia were considered unpromising in terms of the possibility of having genetic offspring. A breakthrough in the rehabilitation of patients with severe pathospermia and azoospermia was the introduction of the IVF-TESA - ICSI technique. In this regard, it became necessary to reconsider the attitude to patients with azoospermia as unambiguously unpromising. However, empirical treatment of patients with azoospermia is unacceptable and not justified. This category of patients is shown testicular biopsy only in the absence of microdeletions of the entire AZF, AZFa or AZFb regions. Because in $46, \mathrm{XX}$ men, as well as in patients with complete deletions of AZF, Azfa, AZFb or AZFb+c, severe disorders of spermatogenesis are detected up to Sertoli cell-only syndrome, which indicates that it is impossible to obtain spermatozoa [4-7]. Therefore, with these deletions, a negative result is predicted during testicular biopsy and TESA procedure. Less severe in terms of reproduction are deletions that completely cover the AZFc region, which account for $75-80 \%$ of all complete AZF deletions [7]. With complete deletion of the Azfc region, successful sperm extraction is predicted. At the same time, there is a fairly clear dependence of the degree of spermatogenesis disorder on the size and localization, i.e. on the type of deletion [7]. This is an important prognostic value in relation to the possibility of reproduction and obtaining sperm suitable for artificial insemination. TESA (testicular sperm aspiration) is the" Gold standard " of Reproductology for both non-obstructive and obstructive azoospermia. In this procedure, sperm is obtained by aspiration of testicular tissue through a thin needle inserted into the testicle through the skin. This method allows you to get a sufficient volume of sperm for work, is not traumatic and does not require special equipment. [10]. Spermatozoa obtained during puncture can be cryopreserved for subsequent ICSI cycles. Cryopreservation of spermatozoa allows you to avoid unnecessary stimulation of a woman's ovaries and additional surgical intervention if the first attempt of ICSI was not successful. This allows you to eliminate the factor of loss of sperm quality, avoid the need to synchronize manipulations with another partner, and completely eliminate the risk of useless superovulation induction. Thus, sperm puncture using the TESA technique can overcome male infertility in both variants of azoospermia. In this regard, the aim of our study was to assess the frequency of different clinical outcomes of IVF - TESE - ICSI programs in a cohort of infertile couples with azoospermia.

\section{Materials AND Methods}

The study group consisted of 272 married couples in which men were diagnosed with azoospermia.

The criteria for selecting patients were:

1) male infertility factor-azoospermia.

2) the absence of karyotype abnormalities, deletions of the Azfa, $\mathrm{AZFb}$ or $\mathrm{AZFb}+\mathrm{C} \mathrm{Y}$ chromosome according to the survey data; the absence of a history of cryptorchidism, chemotherapy, radiation therapy, taking steroid hormones, anabolics, being in the radiation zone, or the presence of testicular malignancies.

Theexamination of men included:clinical, andrological, and genetic. All patients underwent genetic analysis (karyotype, analysis of AZF region microdeletions, analysis of CFTR cystic fibrosis gene mutations) after full consultation. Spermological analysis of the ejaculate was performed according to the standard procedure recommended by WHO (2010) [11]. When analyzing the ejaculate, the following parameters 
were evaluated: volume, color, consistency, sperm concentration in $1 \mathrm{ml}$ of ejaculate, degree of mobility, and quantitative analysis of pathological forms of sperm according to Kruger.

\section{RESULTS}

We examined 272 married couples with male infertility factor-azoospermia, who had TESA procedure between January 2016 and July 2020. In 73\% of patients after the TESA procedure, spermatozoa were obtained. In $27 \%$ of patients, no sperm was obtained.

Figure 1 shows the age distribution of men who had azoospermia and a negative result during the TESA procedure (no sperm was found).

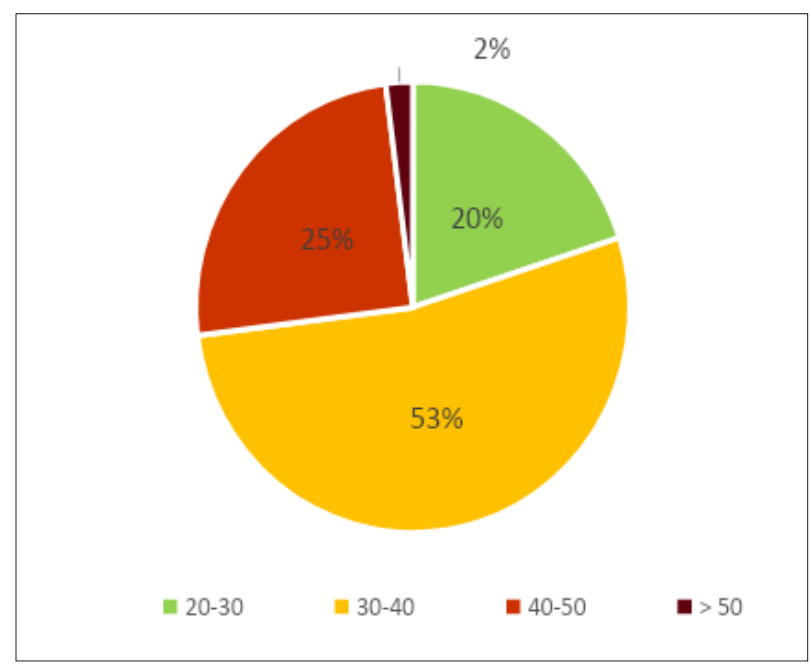

Fig1. The age distribution of men who had azoospermia and a negative result during the TESA procedure (no sperm was found).

It can be seen that $73 \%$ of this category of men were under 40 years of age, $25 \%$ of men were middle - aged (from 40 to 50 years), and only $2 \%$ of men were over 50 years of age. In other words, the negative result of the TESA procedure cannot be explained by agerelated changes in the male body. Figure 2 shows the distribution diagram for the results (outcomes) of IVF - TESA -ICSI programs in couples with infertility, men who had azoospermia and a positive result during the TESA procedure. At the same time, by the result of these programs, we mean not only the result of fresh embryo transfer, but also the results of cryo transfers of embryos obtained during fertilization of the spouse's oocytes with the husband's spermatozoa found during the TESA procedure. It can be seen that clinical pregnancy occurred in $47 \%$ of patients in this category, but $17 \%$ of them had a miscarriage. This can be explained by the high percentage of abnormalities in the spermatozoa of men with azoospermia. 33\% of the patients did not become pregnant, in $9 \%$ all embryos were anomalous, and $11 \%$ of patients had a diagnostic TESA.

At the same time, $25 \%$ of patients with non-interrupted clinical pregnancy-pregnancy occurred after a fresh embryo transfer, in $45 \%$ of patients this category the pregnancy after the first cryo embryo transfer, 9\% after 2 cryo embryo transfer, 13\% after the third cryo embryo transfer, and 5\% after 4 cryo embryo transfer. Thus, in the majority of patients $(70 \%)$ of this category, pregnancy occurred after fresh embryo transfer or after first cryo embryo transfer .

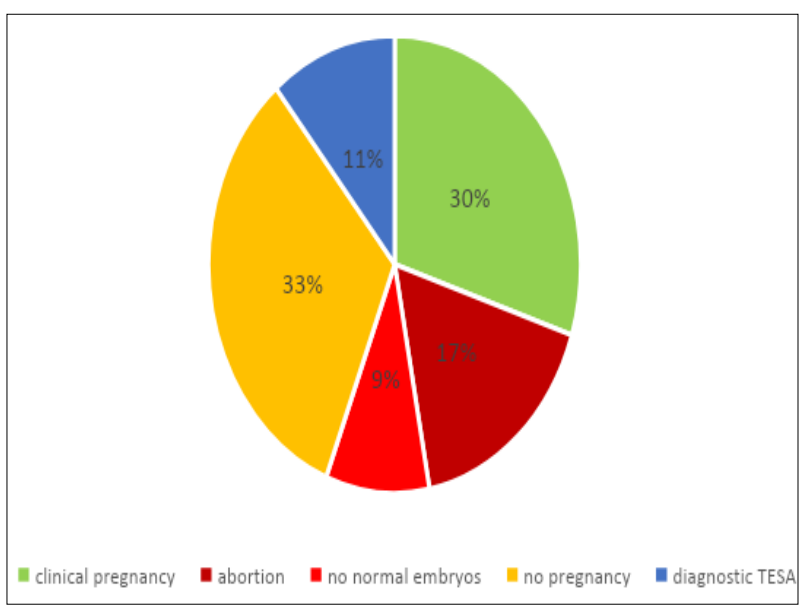

Fig2. Distribution by results (outcomes) of IVF-TESE ICSI programs in couples with infertility

In addition, 60 patients in the examined group underwent a repeated TESA procedure. It was shown that during the repeated TESA procedure, $89 \%$ of patients who were found to have spermatozoa for the first time were also found to have spermatozoa. No spermatozoa were detected in $8 \%$ of patients during the repeated TESA procedure. If the patient was not found to have spermatozoa during the first TESA procedure, then they were not found to have spermatozoa during further procedures TESA.

\section{Discussion}

In recent years, the range of assisted reproductive technologies has expanded significantly, and many couples with infertility, even with severe forms of infertility, have been able to have their own children. However, these patients need medical 
Overcoming Infertility in Men with Non-Obstructive and Obstructive form of Azoospermia thought the Procedure Tesa

and genetic examination both to identify the cause of pathozoospermia, differential diagnosis of male infertility, confirmation/verification of the diagnosis, and to assess the possibility of reproduction (using assisted reproductive technologies), predict its success and determine the genetic risks in offspring. For all patients with azoospermia, a very accurate history is required to determine the potentially correctable factors that led to infertility. Some men may have cryptorchidism. Hormonal changes, for example, in men with NOA are usually characterized by increased levels of serum FSH and reduced levels of testosterone [10]. Before further intervention, it is recommended to conduct comprehensive treatment, including surgical treatment of cryptorchidism, expressed varicocele, and hormonal disorders. To determine the success criteria for subsequent TESA surgery, some authors recommend Doppler mapping of the testicles and starting a biopsy in the area of maximum perfusiot. [12]. A high level of FSH is not a reason for refusing to perform the TESA procedure in men with NOA. Since neither the level of FSH nor the volume of the testicles, according to many researchers, are not associated with the effectiveness of the TESA procedure [13-14].

In connection with the above, the search for new moleculargeneticmarkersofresidual spermatogenesis is an extremely promising area of research. For example, it was found that ESX 1 mRNA is detected in all cases of the presence of germ cells in seminal tubules. A useful addition to the usual prognosis parameters is the expression of the VASA gene in the testicles [10].

Genetic testing to determine the cause of reduced spermatogenesis can provide important prognostic and diagnostic information. Preliminary (before surgery) testing for microdeletions of the AZF region of the $\mathrm{Y}$ chromosome and karyotype analysis makes it possible not only to determine the cause of azoospermia, but also to predict the effectiveness of testicular biopsy. As mentioned earlier, the prognosis of success of the TESA procedure is favorable for men with AZFc deletions, while the effectiveness of sperm search in men with complete deletion of AZFa or AZFb (or $\mathrm{AZFb}+\mathrm{c}$ ) is zero. But it should be remembered that deviations in the male karyotype, as well as the use of spermatozoa with deletions in the AZF region in the case of boys ' birth, in most cases leads to the transmission of this pathology to the offspring. In addition, patients with various forms of azoospermia have genetic features of gametes. L. Rodrigo et al., revealed an increase in the frequency of chromosomal aberrations $(13,18,21, X$ and $Y)$ in sperm samples from patients with azoospermia. There was an increase in sex chromosome dysomy - in $29 \%$ of patients with OA; men with NOA were more likely to have diploidyin $54 \%$ of cases and dysomy of the 13th, 21st and sex chromosomes compared to the control group [15]. Thus, a number of authors have shown that the testicular spermatozoa of patients with azoospermia often show chromosomal abnormalities. However, if a girl is born, if PGD (preliminary genetic diagnosis) is performed, the child may be completely healthy. In addition, experts note that men with detected disorders in the AZF region of the $\mathrm{Y}$ chromosome show a progression of spermatogenesis disorders [7]. Therefore, all patients with these disorders are recommended to use cryopreservation of the obtained material.

Thus, genetic factors are one of the main causes of infertility [4-7]. However, despite the fundamental importance of genetic factors for reproduction, often the causes of male and female infertility can not be established. This is due to the insufficient use of new molecular cytogenetic and molecular genetic research methods in practical medicine [7, 16-17]. The advantages of full-exome sequencing (FES) are the creation of new approaches in molecular genetic diagnostics based on the analysis of all possible genes, including recently discovered ones that are not present in clinical (targeted) gene panels. All published results of newly discovered mutations associated with male infertility, obtained using NGS technologies, indicate that its use significantly increases the effectiveness of finding out the molecular and genetic causes of this disease. [16-17]. We believe that FES can be recommended as an important research method for the molecular genetic diagnosis of male infertility.

\section{ConClusion}

In recent years, the range of assisted reproductive technologies has expanded significantly, and many couples with infertility, even with severe forms of infertility, have been able to have their own children. We examined 272 married couples with male infertility factor-azoospermia, who had TESA procedure between January 2016 and July 2020. In 73\% of 
Overcoming Infertility in Men with Non-Obstructive and Obstructive form of Azoospermia thought the Procedure Tesa

patients after the TESA procedure, spermatozoa were obtained.

It can be seen that clinical pregnancy occurred in $47 \%$ of patients in this category, but $17 \%$ of them had a miscarriage. This can be explained by the high percentage of abnormalities in the spermatozoa of men with azoospermia. Thus, in the majority of patients $(70 \%)$ of this category, pregnancy occurred after fresh embryo transfer or after first cryo embryo transfer .

It was shown that during the repeated TESA procedure, $89 \%$ of patients who were found to have spermatozoa for the first time were also found to have spermatozoa.

No spermatozoa were detected in $8 \%$ of patients during the repeated TESA procedure. If the patient was not found to have spermatozoa during the first TESA procedure, then they were not found to have spermatozoa during further procedures TESA.

It is shown that the negative result of the TESA procedure cannot be explained by age-related changes in the male body, since most men in this category were under 40 years of age.

To identify the cause of pathozoospermia and assess the possibility of assisted reproductive technologies, predict their success and determine the genetic risks in offspring, it is necessary to conduct a medical and genetic examination of patients. Further improvement of the effectiveness of IVF-TESA-ICSI programs involves further evaluation of the role of non-invasive techniques (such as dopplerography) in identifying the foci where sperm are most likely to be detected, as well as the search for adequate molecular genetic predictors of successful sperm extraction.

\section{REFERENCES}

[1] Foresta C., Ferlin A., Gianaroli L., Dallapiccola B. Guidelines for the appropriate use of genetic tests in infertile couples. Eur J Hum. Genet 2002;10:5:303-312.

[2] Chernykh V.B. Cystic fibrosis gene and fertility disorders in men. Andrologiya i genital'naya khirurgiya = Andrology and Genital Surgery 2010;(4):23-31. (In Russ.)

[3] Matsumiya K., Namiki M., Takahara S. et al.
Clinical study of azoospermia. Int J Androl 1994;17(3):140-142.

[4] Raman J.D., Schlegel P. Testicular sperm extraction with intracytoplasmic sperm injection is successful for the treatment of nonobstructive azoospermia associated with cryptorchidism. J Urol 2003;170(4 Pt 1):1287-90.

[5] Aziz N. The importance of semen analysis in the context of azoospermia // Clinics (Sao Paulo), 2013. Vol. 68. Suppl 1. P. 35-38.

[6] Esteves SC, Miyaoka R, Agarwal A. An update on the clinical assessment of the infertile male // Clinics (Sao Paulo), 2011. Vol. 66. P. 691-700.

[7] Chernykh V.B., Rudneva S.A., Sorokina T.M., Shileyko L.V., Kurilo L.F., Ryzhkova O.P., Chukhrova A.L., Polyakov A.V. Characteristics of spermatogenesis in infertile men with the AZFc region deletions. Andrology and Genital Surgery. 2014;15(2):48-57. (In Russ.)

[8] Vogt P.H., Edelmann A., Kirsch S. et al. Human Y chromosome azoospermia factors (AZF) mapped to different subregions in Yq11. Hum Mol Genet 1996;5(7):933-43.

[9] Chernykh V.B., Rudneva S.A., Sorokina T.M., Shileyko L.V., Ostroumova T.V., Ermolaeva S.A., Kurilo L.F., Ryzhkova O.P., Bliznets E.A., Chukhrova A.L., Polyakov A.V. An influence of androgen receptor (AR) gene CAG-polymorphism on spermatogenesis in infertile men. Andrology and Genital Surgery. 2015;16(4):55-61. (In Russ.)

[10] I.I. Vityazeva In vitro fertilization with intracytoplasmatic sperm injection in treatment of infertility in men with non-obstructive azoospermia: microdissection technique of sperm extraction from testicular tissue. // Andrology and Genital Surgery. 2014;15(2):622. (In Russ.)

[11] WHO Laboratory manual for the examination and processing of human semen. Trans. from English by N.P. Makarova. Scientific ed. by L.F. Kurilo. 5th ed. Moscow: Kapital Print, 2012. (In Russ.)

[12] Foresta C., Garolla A., Betella A. et al. Doppler ultrasound of the testis in azoospermic subjects as a parameter of testicular function. Hum Reprod 1998;13(11):3090-3. 
Overcoming Infertility in Men with Non-Obstructive and Obstructive form of Azoospermia thought the Procedure Tesa

[13] Tsujimura A. Microdissection testicular sperm extraction: prediction, outcome, and complications. Int J Urol 2007;14(10):883-9.

[14] Hibi H., Ohori T., Yamada Y. et al. Probability of sperm recovery in nonobstructive azoospermic patients presenting with testes volume less than $10 \mathrm{ml} / \mathrm{FSH}$ level exceeding $20 \mathrm{mIU} / \mathrm{ml}$. Arch Androl 2005;51(3):225-31.

[15] Rodrigo L., Rubio C., Mateu E. et al. Analysis of chromosomal abnormalities in testicular and epididymal spermatozoa from azoospermic ICSI patients by fluorescence insitu hybridization. Hum Reprod 2004;19(1):118-23.

[16] Rebrikov D. V., Korostin D. O., Shubina E. S., Ilinsky V. V. NGS: high-performance sequencing. Under the General editorship of D. V. Rebrikov. 2nd ed. M.: Binom. Laboratory of knowledge, 2015.

[17] Worthey E.A. Analysis and annotation of wholegenome or whole-exome sequencing-derived variants for clinical diagnosis. Curr Protoc Hum Genet 2013;79:Unit 9.24.

Citation: Rudneva S. A., Apryshko V. P, et al. Overcoming Infertility in Men with Non-Obstructive and Obstructive form of Azoospermia thought the Procedure Tesa. Archives of Urology. 2020; 3(2): 29-34.

Copyright: (C) 2020 Rudneva S. A., Apryshko V. P, et al. This is an open access article distributed under the Creative Commons Attribution License, which permits unrestricted use, distribution, and reproduction in any medium, provided the original work is properly cited. 